\title{
Research and Design of Autism Smart Diagnosis Information System Based on Chinese Children's Facial Expression Data and Deep Convolution Neural Network
}

\author{
Wang Zhao \\ School of Information Management, Wuhan University \\ 199223263@qq.com
}

\begin{abstract}
In this paper, the standard facial expression database FER2013 and CK + are used as the main training samples for autism diagnosis model.The facial expression image data of 16 Chinese children were collected as supplementary training samples.We use deep convolution neural network VGG19 and Resnet18 artificial intelligence algorithms to research and develop an smart information system for the diagnosis of autism through facial expression data.Ten normal children and ten autistic children were recruited for the comparative test to verify the accuracy of the system.After testing, the accuracy of facial expression recognition of this system reaches $81.4 \%$.This research is based on the actual business needs of the hospital. The system can diagnose autism as early as possible, and promote the early treatment and rehabilitation of patients, thereby reducing the economic and mental burden of patients. Therefore, this smart information system has good social benefits and application value.
\end{abstract}

\section{Introduction}

Facial expression recognition is an important social cognitive skill. Emotions are expressed by facial expressions. Therefore, recognition and understanding of facial expressions is the basis of communication and interpersonal relationships with others. Abnormal expression is a prominent manifestation of autism, and it is also one of the criteria for the diagnosis of autism. Doctors can diagnose autism by observing children's abnormal facial expressions.

Autism, also known as autism or autism disorders, is a representative disease of generalized developmental disorders. In recent years, the incidence of autism in children has become higher and higher, experiencing a transition from rare diseases to epidemics.At present, research on autism is still in its infancy at home and abroad, and research methods and tools are still developing.

The main symptoms of autism are social and interpersonal disorders, language retardation, repetitive behavior and sensory dysfunction. For autistic patients, it is difficult to correctly identify and interpret facial emotions. Their emotional expression is different from that of ordinary people. They can not correctly perceive and understand some basic facial emotions, such as anger [1].

At present, the diagnostic methods for autism spectrum disorders include: traditional standard DSM-IV-TR [2] and ICD-10 [3], various autism diagnostic assessment scales such as "Child Autism Rating Scale (CARS)", "Autism Children ABC Test Scale" and Autism Behavior Rating Scale, and questionnaire interviews [4].Most of these methods rely on doctors'direct observation of the patient's expression, speech and behavior based on their experience.Diagnostic results are easily disturbed by external factors such as hospital level, physician's subjective level, patient's education level, age and so on. There are relatively large subjective factors, resulting in a certain degree of missed diagnosis and misdiagnosis.It takes about 1 to 2 hours for each autistic patient to diagnose, so doctors have a lot of work to do. The best period of treatment for autistic patients is before the age of six. Early diagnosis is of great significance for the rehabilitation of autistic patients.

The purpose of our research and design is to train facial expression recognition model and establish a facial expression recognition system based on normal expression to verify abnormal expression. The system can detect the facial expression of autistic children and judge the difference between autistic children and normal children. 
In this study, FER2013 and CK + were used as the main facial expression training samples. At the same time, we collected the facial expression image data of 16 Chinese children as a supplementary sample of facial expression.With the help of VGG19 and Resnet18 algorithm models of deep convolution neural network, according to the hospital autism diagnosis scale and diagnosis process, this paper studies and designs a smart information system for the diagnosis of autism by facial expression data. After the actual test of recruiting testers, the recognition rate of the system is $81.4 \%$. It can effectively distinguish whether the expression of children is normal or not. It provides a practical information system for the diagnosis of autism.

The autism smart diagnosis information system designed in this study has the following important significance:

(1)Autism can be diagnosed as early as possible by using this system. The best time to treat autism is before the age of 6 . The earlier the diagnosis of autism is made, the less the treatment will cost and the higher the probability of recovery. Early diagnosis is of great significance to alleviate the burden of autism patients and society. The system can be published in the form of APP or web pages and disseminated through the Internet. The system can be installed and used on different devices, such as computers, mobile phones, tablets, etc. It has good applicability. The system can easily diagnose autism and gain time for the early treatment of autistic patients, especially those with underdeveloped medical conditions.

(2)It can make the diagnosis of autism more objective. The whole diagnosis process is completed by the system. Because artificial intelligence technology is used to recognize facial expressions without human intervention, the diagnosis results are objective and accurate.

(3)Reduce the intensity of doctors'work. Before the system was used, it took an hour for doctors to diagnose an autistic patient. By using this system, doctors can save a lot of time and pay attention to the treatment of autism.

(4)This system uses FER2013 and CK+ as the main facial expression training samples. These two expression databases contain facial expression data from people in different countries and regions, so this system is suitable for the diagnosis of autism for children and adults all over the world. We also collected facial expression images of 16 Chinese children as supplementary samples, increasing the number of Asia-Pacific children's expression data in the training samples. The aim is to increase the sensitivity of facial expression recognition in Asia Pacific children and further improve the recognition rate of children in Asia Pacific.

(5)The system is designed according to the actual business of the hospital. This study adopted the recommendations of several doctors, designed according to the actual needs of doctors for the diagnosis of autism. Although there are some papers on the diagnosis of autism through facial expressions at home and abroad, there are still relatively few autism diagnosis systems that can be used.

(6)This paper uses the latest deep learning technology to improve the accuracy of facial expression recognition. Previous conventional techniques and methods have lower facial expression recognition rates. In recent years, with the development of artificial intelligence technology and the increase of computer computing speed, convolutional neural network has greatly improved the accuracy of facial expression recognition, which is the innovation of this research in technology.

\section{Facial expression recognition process and recognition technology selection}

\subsection{Facial expression recognition process}

The process of facial expression recognition includes two stages. As shown in Figure 1: One is the training process and the other is the recognition process. The training and recognition process can be divided into three parts: the pretreatment of facial expression images, the extraction of facial expression features and the classification of facial expressions. The training process is to train the model in order to achieve the purpose that the model can be used. The recognition process is to recognize and classify the facial expression of the test image [5].

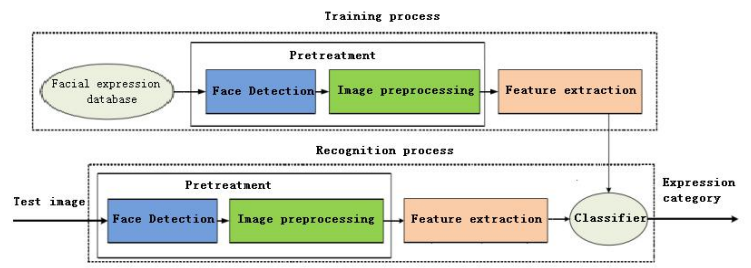

Figure 1. Facial expression recognition process

The two stages of facial expression recognition process include the following processes: First, face detection is carried out on the image in the expression database, including the location, alignment and 
clipping of the face area. This is the basis of the follow-up process. Only when the facial expression area is accurately obtained, the following series of work will be more accurate. After the face area is detected, the image needs to be preprocessed. In order to eliminate the noise caused by the acquisition equipment and environment, and avoid the interference in feature extraction. Then it is the feature extraction step, which aims to extract the features that can represent the essence of expression from the preprocessed facial images. In this process, in order to avoid the high dimension of feature extraction and affect the efficiency of the algorithm, we need to reduce the dimension of extracted features in order to extract the most representative expression features. Finally, the extracted facial features are classified to determine which type of facial expression is.

\subsection{Selection of facial expression recognition technology}

Facial expression recognition technology mainly includes traditional machine learning technology and deep learning technology. The two technologies have similarities and different characteristics.

2.2.1 Traditional machine learning technology. Facial expression recognition algorithm based on traditional machine learning includes three steps: image preprocessing, facial expression feature extraction and feature classification.

Firstly, for the convenience of feature extraction, it is necessary to preprocess the image, which can effectively avoid the interference of various noises and leave the key information needed by the face. The pretreatment process includes image gray processing, face alignment, face size tailoring, data enhancement, brightness and pose normalization, etc [6].

Secondly, the traditional feature extraction methods include directional gradient histogram feature, Gabor filter feature, local directional pattern feature and enhanced local binary algorithm.Because these methods are artificial design, time-consuming and laborious, and have certain limitations.Because traditional machine learning have better effect in feature extraction only in small sample image set, so most of the current studies are based on deep learning feature extraction method.

2.2.2 Deep learning technology.Facial expression recognition algorithm based on deep learning also needs image preprocessing. The difference is that it often combines feature extraction and feature classification into an Endto-End model, which greatly simplifies the process of facial expression recognition.In addition to Endto-End learning, deep learning algorithm can be used to extract facial expression features, and then other independent classifiers can be used.For example, SVM or random forest algorithm is used to process the extracted features and classify them.

In this paper, we construct a facial expression recognition model based on deep learning technology, extract facial expression feature data of children and classify them into groups, so as to diagnose autism.

\section{Autism and facial expression diagnosis}

\subsection{Autism and its development}

Autism is a neurodevelopmental disorder, which is collectively referred to as autism spectrum disorder [7].Since Kanner, an American child psychiatrist, first reported autism in 1943, the incidence of autism has risen rapidly worldwide.In the $1980 \mathrm{~s}$, about 3-5 out of every 10,000 people suffered from the disease, while in $2000,6.7$ out of every 1,000 children suffered from the disease [8].According to the National Center for Health Statistics, the probability of autism among children aged 3-14 in the United States reached $2.76 \%$ in 2016 [9].

There is no statistical survey on autistic children in China.However, according to the data of the Report on the Development of China's Autism Education and Rehabilitation Industry II, the number of people with autism in China is estimated to exceed 10 million, of which 2 million are autistic children. At the same time, it is growing at the rate of nearly 200,000 annually [10].

Autism brings serious financial burden to both society and family. Families with autistic children, on the one hand, spend a lot of time caring for their children, working hours are reduced so that work income is reduced.On the other hand, the cost of family rehabilitation treatment for autistic children is huge, which increases the family's financial burden [11].According to the survey on the occupational and economic burden of preschool autistic children's families, $33 \%$ of parents of autistic children reported that their caregiving problems seriously affected their careers, and their annual income was significantly lower than that of ordinary families, with an average loss of income of 30,957 yuan per year. Meanwhile, the average annual cost of autistic children's families for children's education and training is significantly higher than that of ordinary families [12]. The society 
and the government also need to invest a lot of money in the rehabilitation education of autistic children. At the same time, autism also brings high subjective load and depression to the families of patients, which has a negative impact on their quality of life $[13,14]$.It can be seen that the incidence of autism in children is relatively serious, and the harm to society and family is enormous.

\subsection{Diagnosis of autism through facial expressions}

3.2.1 Facial expression recognition disorder. The autistic children have facial expression recognition obstacles, which are mainly manifested in their inability to recognize facial expressions [15].It is easy to distinguish autistic children from normal children by observing their facial expressions.Therefore,We extract facial expression feature and use facial expression recognition technology to distinguish normal children and autistic children.

3.2.2 The principle of diagnosing autism through facial expressions.A large number of studies have pointed out that autistic patients have deficiencies in facial expression recognition and understanding. This is the core source of impaired social function in autistic patients [16]. Autistic children are more difficult to identify other people's emotional behavior, and it is difficult to make appropriate judgment and response [17]. Overseas research on facial expression recognition ability of autistic patients has been carried out not only in children, but also in adults. Most studies believe that the ability of facial expression recognition of autistic patients is low. BaronCohen et al.used standard facial expression maps to study the recognition of different emotional types in autistic adults [18]. It was found that autistic adults had better recognition of some basic facial expressions, such as happiness, but relatively complex facial expressions such as surprise recognition were difficult to recognize.

At present, the main diagnostic criteria of autism are: IDC-10, DSM-IV, the Autism Child Behavior Scale (ABC scale), the Children Autism Rating Scale (CARS scale), and the Clancy Behavior Scale (CABS scale) [19].

After consulting a large number of literatures and investigating the actual situation of the hospital, now the hospital mainly uses KBS (filled by parents), ABC (filled by parents), CARS (filled by doctors) to diagnose autism. After a detailed review of the test items of the three scales, these scales all contain the test items to judge autism through children's facial expressions. There were 14 items in the CABS scale, of which the seventh item was inexplicable laughter and the tenth item was not looking at each other's face. Avoiding eye contact was related to expression. There were 57 items in the $\mathrm{ABC}$ scale, of which the seventh item was non-communicative smile, the seventeenth item did not respond to other people's facial expressions, and the twenty-fourth item was active avoidance of eye contact with others. Fifteen items of the CARS scale, the third of which is emotional response, pleasure and unhappiness, and interest, are expressed by changes in facial expression and posture. These scales basically include the items of autism detection by children's facial expressions, which shows that the diagnosis of autism can be more accurate by facial expressions. With the progress of artificial intelligence technology, facial expression recognition technology can objectively and effectively reflect the mental health of children, and can be used in early diagnosis of autism [20].

We also communicated with doctors of Hubei Maternal and Child Health Hospital, Wuhan Children's Hospital and Guangzhou Women and Children's Medical Center many times, and actually checked the process of using the above autism diagnostic scale to diagnose children. The doctor observes the tester's reaction to determine whether the tester is autistic after requesting the tester to make the corresponding expression. Doctors point out that facial expression is an important part of autism diagnosis. In terms of system design, they put forward requirements and suggestions for the process of diagnosing autism through facial expression.

\section{Research and development of autism diagnosis information system}

\subsection{Facial expression database selection}

The facial expression databases in this study mainly come from two public facial expression databases $\mathrm{CK}+$ and FER2013. In addition, 16 Chinese children's facial expression data were collected as supplementary samples. The two public facial expression databases are standard and international, and have been widely used, including facial expression data of adults and children. Each sample in the database contains seven facial expressions: angry, disgust, fear, happy, sad, surprise, and neutral. Because children's facial expressions are different from adults, in order to improve the recognition rate of children's facial expressions, we 
collected facial expression data of 16 children aged 5 to 8 in China. Seven facial expressions were collected from each child. We combine Chinese children's facial expression data and public facial expression database as our system's facial expression database.

4.1.1 FER2013 facial expression database.The reason for choosing FER2013 expression database is that it has more samples and is more mature than other facial expression databases. It has advantages in model training. At the same time, it has been used in many studies.

There are 35887 facial images in the facial expression database, and there are seven facial expression types: angry, disgust, fear, happy, sad, surprise and neutral. The resolution of each image is $48 * 48$ pixels. As shown in Figure 2.

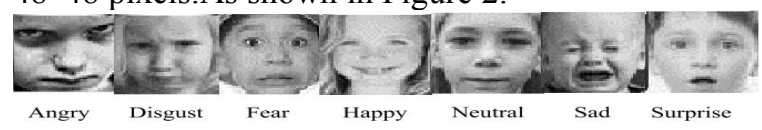

Figure 2. FER2013 facial expression database

4.1.2 CK+ facial expression database.CK + facial expression database was selected because it was collected in the laboratory, so its accuracy is relatively high [21].

The facial expression database was collected under laboratory conditions. It includes African Americans, Asians and South Americans. The resolution of each image is $640 * 480$ pixels. It contains 593 expression sequences of 123 people, $69 \%$ of whom are female and $31 \%$ are male. It includes seven types of facial expressions: anger, contempt, disgust, fear, happy, sadness, surprise. As shown in Figure 3.

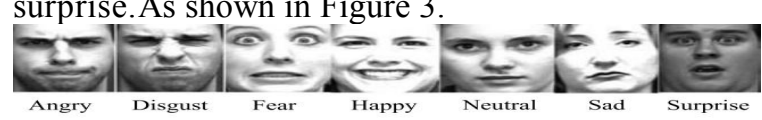

Figure 3. CK+ facial expression database

4.1.3 Facial expression data of Chinese children.At present, the mature facial expression databases at home and abroad are mainly based on adult male or female facial expression images. Therefore, it is urgent to establish a facial expression database for children.

Facial images of children are quite different from those of adults. Children have rounder faces, larger eyes and less prominent bones. Because of these differences, children's facial features are less obvious and more difficult to recognize than adults. Because of the particularity of children, it is very difficult to collect children's facial images. In order to improve the recognition rate of children's facial expressions, we cooperated with Amy Education School in Zhengzhou. Sixteen healthy children volunteers were recruited to collect facial expression data. Each of them collected seven kinds of expressions, totaling 112 pictures. These children are between 5 and 8 years old, including 8 boys and 8 girls. The acquisition environment is quiet and there is no external interference. Highdefinition cameras are used to collect facial expression images, which are processed professionally. Before collecting facial expression data, parents have been informed of the purpose of collecting facial expression data. After questioning with parents, all the children who participated in the collection of facial expression data had no history of autism.

We loaded the facial expression data into the training sample library. The purpose of collecting Chinese children's facial expression data is to increase the number of Chinese children's facial expression samples in training samples.This can improve the sensitivity of the system to AsianPacific children's facial expressions, and further improve the recognition rate of Asian-Pacific children's facial expressions. The collection process and the collected children's facial expression data are shown in Figure 4.

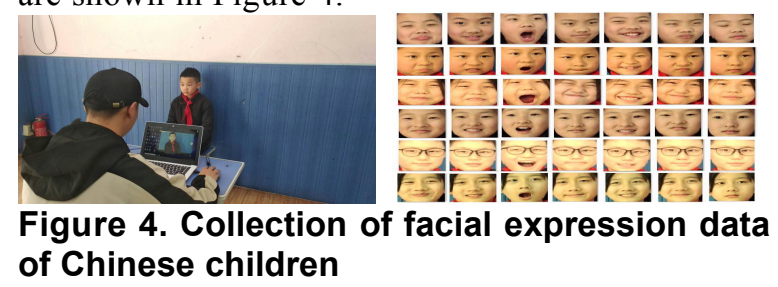

\subsection{Network topology}

According to the network environment and equipment of the information service platform, the network topology can be divided into four levels. The network topology diagram is shown in Figure 5.

The first layer is the application layer, which consists of users, computers and various smart devices. Smart devices include smart tablet computer, smart phones and other electronic devices. Users access and use the information service platform through computers and various smart devices.

The second layer is the communication layer, mainly based on the Internet network environment, providing access channels for users and systems.

The third layer is the application server layer, which is composed of firewall and application server, and has an ontology display system for autism. The application server manages various business functions, handles various business requests 
submitted by users, and can access the database server for various data exchange.

The fourth layer is the database server layer, which stores all kinds of data and knowledge resources of the information service platform.

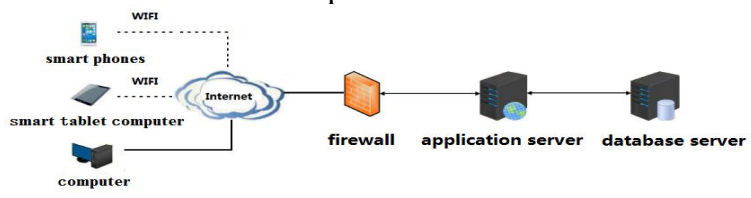

Figure 5. Network topology diagram

\subsection{System architecture}

The smart diagnosis system of autism adopts Client/Server architecture. The client includes different versions of programs suitable for computers and smartphones. The system architecture diagram is shown in Figure 6.

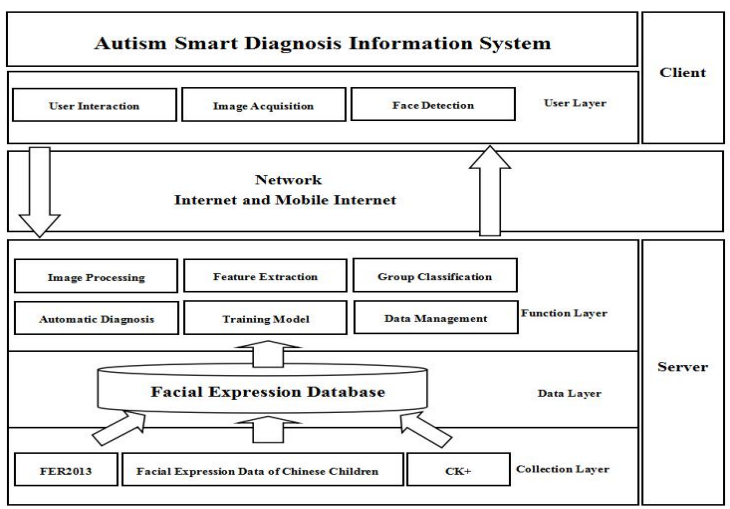

Figure 6. System architecture diagram

The client includes three main modules: user interaction, image acquisition and face detection.User interaction module is responsible for humancomputer interaction. According to the requirements of the Autism Diagnostic Scale, users who diagnose are required to make appropriate expressions and feedback by prompting pictures and voice guidance.Through the camera, the image acquisition module can dynamically capture facial expression images. At the right time, the system will collect facial expression images and transmit them to the face detection module.Face detection module recognizes the valid face features, and compresses the image and transfers it to the server through the Internet or mobile Internet.

The server includes six main modules: image processing, feature extraction, group classification, automatic diagnosis, training model and data management.The image processing module can receive the expression image transmitted by the client, and then process the expression image and transfer it to other modules on the server side.The feature extraction module receives the facial expression images provided by the image processing module, and extracts the facial expression features. The group classification module is responsible for group classification, and correctly classifies the expression images into the most matching expressions among the seven kinds of expressions. The automatic diagnosis module gives the diagnosis of autism by comparing the facial expressions that the tester is required to imitate and the facial expressions that the tester actually makes.Model training module is the core module of the system, which is responsible for recognizing and processing the newly collected facial expression images. The data management module mainly manages facial expression data, including storing and reading facial expression images transmitted by the client.

The system server stores facial expression feature files, which are formed by feature extraction of facial expression database. The expression feature file is HDF5 file format. The expression recognition system running on the server can read the expression feature file at any time. If new facial expression samples are collected, the model can be retrained and the facial expression feature files can be updated.

The client collects the tester's facial expression data by high definition camera, and transmits the facial expression data to the server by JSON file according to TCP communication protocol. The facial expression recognition system running on the server processes the collected facial expression data, and then feeds the recognition results back to the tester through the network, and stores the recognition results and facial expression data in the server database. Facial data and diagnostic system are stored on a server, and the recognition results and facial data are stored in the SQL Server database. The diagnostic system reads data from the database through SQL structured query language. The response time of the whole database operation and communication process should not exceed 5 seconds.

\subsection{Deep learning framework}

4.4.1 VGG-19 model.Researchers from Oxford University and the Google Deep Brain Group have jointly developed the convolutional neural network VGG.VGNet consists of 11, 13, 16 and 19 layers of neural networks [20].VGNet constructs 16-19 layers of neural networks by stacking small convolution cores of $3 \times 3$ and maximum pooling layers of $2 \times 2$ 
repeatedly.VGGNet has strong scalability and greatly reduces the error rate when extending. When migrating to other image data, it has good generalization ability and simple structure.

4.4.2 ResNet-18 model.ResNet was proposed by Kaiming $\mathrm{He}$ and others of Microsoft Research Institute. They have successfully trained 152 layers of neural networks by using ResNet Unit.The structure of ResNet can accelerate the training of the neural network, and the accuracy of the model has been greatly improved.

4.4.3 Graphic of deep learning framework. The deep learning framework used in this paper for facial expression recognition is shown in Figure 7.

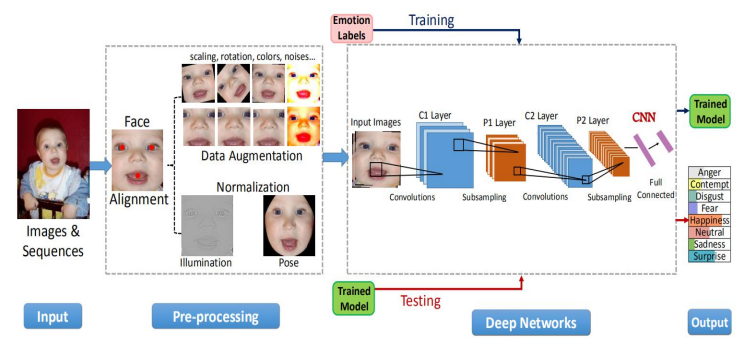

Figure 7. Framework of deep learning

The whole process includes image input, image preprocessing, model building, model training, model testing, and output of expression recognition results.There are two kinds of deep learning algorithms used in this paper: VGG-19 and ResNet18.ResNet-18 solves the problem of network performance degradation caused by the high depth of VGG-19.By training the two models and synthesizing the two convolutional neural network models, the facial expression features of autistic children can be extracted accurately.

4.4.4 Recognition results. Through the trained model, we use some children's facial expressions pictures and videos to test, and get the probability of various expressions and the final prediction results of the model.As shown in Figure 8, the histogram shows the probability of each type of facial expression, and the histogram of maximum probability is the final recognized facial expression.After testing, the recognition rate of children's facial expression reaches $81.4 \%$, which can effectively distinguish whether children's facial expression is normal or not.

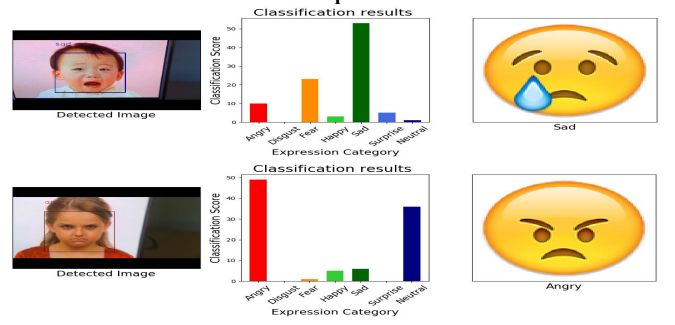

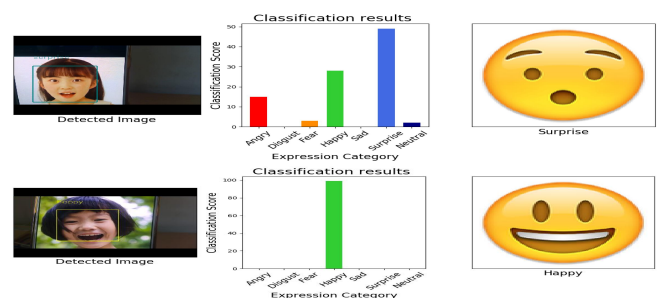

Figure 8. Facial expression recognition results

\section{System validation}

\subsection{Testing environment}

In this study, two kinds of mobile phones, personal computers and servers are selected as test environments. The hardware and software environments are shown in Table 1.

Table 1. Testing environment

\begin{tabular}{|l|ll|l|}
\hline \multicolumn{1}{|c|}{ Testing Equipment } & \multicolumn{2}{|c|}{ Hardware Environment } & \multicolumn{1}{|c|}{ Software Environment } \\
\hline OPPO R17 Mobile Phone & CPU: SDM670 & RAM: 8GB & Android \\
\hline IPhone 8 Mobille Phone & CPU: $A 11$ & RAM: 2GB & IOS \\
\hline Personal Computer & CPU: Intel i7 & RAM: 16GB & Windows 10 \\
\hline Server & CPU: Intel W2133 & RAM: $16 \mathrm{~GB}$ & Windows Server 2019 \\
\hline
\end{tabular}

\subsection{Diagnostic procedure and interface of diagnostic system}

Diagnostic procedures are mainly designed according to the actual process of hospital autism diagnosis. The Autism Diagnostic Scale (ADS) contains a test of the patient's facial expression response. On the one hand, the automatic diagnosis method of this system is designed strictly according to the actual diagnosis method of the hospital. On the other hand, in the process of testing children's facial expressions, some induction methods and tools are added. For example, when testing happy expressions, the system displays happy cartoon smiling faces, plays happy children's songs and animations for situational simulation, and induces children to respond accordingly.

5.2.1 Diagnostic procedure.The diagnostic process is shown in Figure 9. Firstly, the system randomly displays one of the seven kinds of facial expressions for the tester to imitate. The system will prompt the tester to imitate the facial expression by pictures and sounds. For example, the system displays happy cartoon smiling faces, plays happy children's songs, and induces children to make happy expressions. The system displays the same expression example three times, and collects the tester's expression data at the same time. Then the system compares the expression examples and the actual 
collected expression data, and gives the diagnosis results.

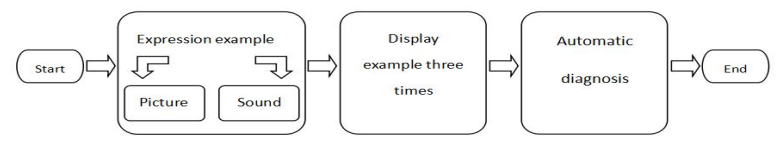

Figure 9. Automatic diagnostic procedure

5.2.2 Interface of diagnostic system. The system diagnostic interface is designed according to the diagnostic process.

Figure 10. A is the main interface of the autism smart diagnosis information system, including system introduction, knowledge introduction of autism and other functions.Figure 10. B is the facial expression that the system prompts the tester to simulate after starting the diagnostic process.Figure $10 . \mathrm{C}$ is the expression analysis after diagnosis.Figure $10 . \mathrm{D}$ is the result given by the system after three diagnoses.

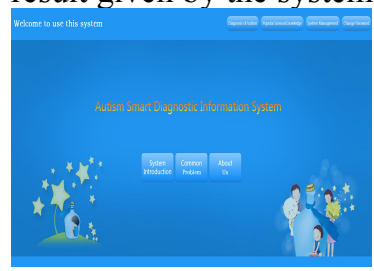

Figure 10. A

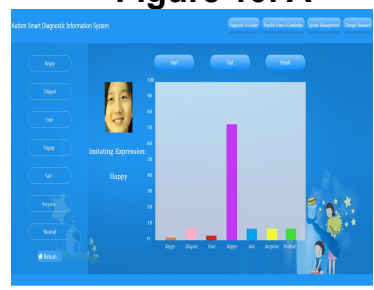

Figure 10. C

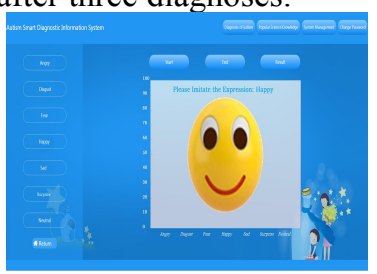

Figure 10. B

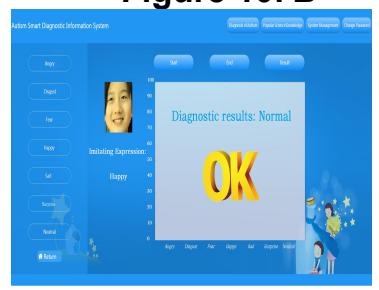

Figure 10. D

\subsection{System testing}

5.3.1. Test sample.We recruited 10 normal children and 10 autistic children and divided them into normal children group and autistic children group. In this way, the grouping can be compared and validated.The accuracy of the system is verified by the actual test of the autism diagnosis information system.

The normal group of children was provided by Amy Education School in Zhengzhou, which cooperated with us. Ten healthy children volunteers were recruited as the normal group for testing. These children were between 5 and 8 years old, including 5 boys and 5 girls. Parents were informed of the purpose and content of the experiment before the experiment. After asking the parents of the children who participated in the experiment, the parents answered that the children who participated in the experiment had no history of autism.
The autistic children were provided by Guangzhou Children's Care Center, which cooperated with us. Ten volunteers of autistic children were recruited as the autistic children group for testing. These children were aged between 3 and 6 years old, including 5 boys and 5 girls. Parents were informed of the purpose and content of the experiment before the experiment. The selected children with autism were diagnosed by a professional physician.

5.3.2. Test environment and process. All the tests were conducted in quiet classrooms without noise and external factors. Through our autism diagnosis information system, each child was prompted by pictures and sounds to imitate seven kinds of facial expressions, and prompted to make corresponding facial responses according to the facial expressions on the pictures. At this time, the camera will capture their facial expressions, and after system analysis,they will be saved in the form of pictures in the computer of the test system.

Figure 11. A is test environment for normal children group.Figure 11. B is test environment for autistic children group.

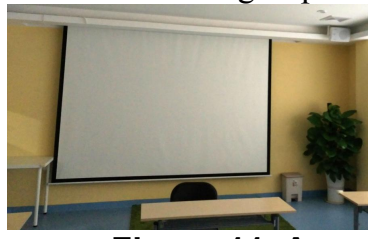

Figure 11. A

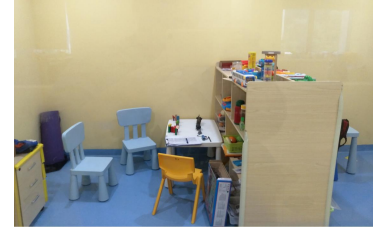

Figure 11. B
5.3.3. Test result. We used the system to test the normal combination and autistic children respectively. Finally, we compared the recognition rate of the two groups.

(1)The normal children group.

From Table 2, the average recognition rate of each expression is angry $80 \%$, disgust $70 \%$, fear $80 \%$, happy $100 \%$, sad $80 \%$, surprise $70 \%$ and neutral $90 \%$.

Test child 1 only had a disgusting expression recognition error, and other facial expression recognition was correct, then the average recognition rate of the seven facial expressions of test child 1 was $85.7 \%$.According to this method, the average recognition rates of the seven expressions from test child 1 to test child 10 were $85.7 \%, 85.7 \%, 85.7 \%$, $57.1 \%, 85.7 \%, 100 \%, 71.4 \%, 100 \%, 85.7 \%, 57.1$, respectively. \%, the average recognition rate is $81.4 \%$.

Judging by the $60 \%$ threshold, there are two test children's facial expression recognition rate is $57.1 \%$. This shows that in real environment, the algorithm of the system is affected by the environment and light, and the accuracy will be affected to a certain extent. However, according to the accuracy of $81.4 \%$, it can basically meet the preliminary diagnostic 
requirements of whether the expression is abnormal or not. In the future, more real samples will be added to further improve the accuracy of the system algorithm.

The experimental results show that the errors mainly concentrate on the expressions of disgust and surprise. The main reasons are as follows:

First,disgust and surprise have only minor local changes in the faces of the two kinds of expressions, and there is no significant distinguishing feature.

Second,some of the participants had little change in the two facial expressions, did not have the obvious features of the corresponding categories, approached neutral expressions, and were easy to confuse.

Table 2. Test results in normal children

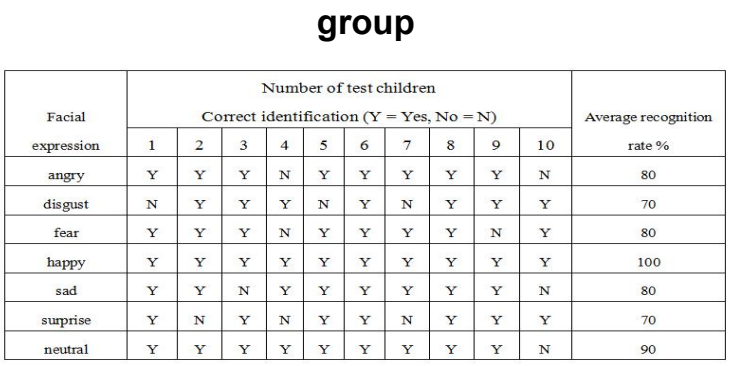

(2)The autistic children group.

From Table 3, the average recognition rate of each expression is angry $50 \%$, disgust $10 \%$, fear $30 \%$, happy $60 \%$, sad $60 \%$, surprise $20 \%$ and neutral $10 \%$.

The average recognition rates of the seven expressions from test child 1 to test child 10 were $28.5 \%, 28.5 \%, 28.5 \%, 42.9 \%, 42.9 \%, 57.1 \%, 28.5 \%$, $42.9 \%, 28.5 \%, 14.3 \%$, and the average recognition rate is $34.3 \%$.

The experimental results show that the recognition rate of happiness and sadness is higher in the seven expressions. Testing children showed difficulty in identifying complex facial expressions such as neutrality and aversion.

Table 3. Test results in autistic children group

\begin{tabular}{|c|c|c|c|c|c|c|c|c|c|c|c|}
\hline \multirow{2}{*}{$\begin{array}{c}\text { Facial } \\
\text { expression }\end{array}$} & \multicolumn{10}{|c|}{$\begin{array}{l}\text { Number of test children } \\
\text { Correct identification }(\mathrm{Y}=\text { Yes, } \mathrm{No}=\mathrm{N})\end{array}$} & \multirow{2}{*}{$\begin{array}{c}\text { Average recognition } \\
\text { rate } \% \\
\end{array}$} \\
\hline & 1 & 2 & 3 & 4 & 5 & 6 & 7 & 8 & 9 & 10 & \\
\hline angry & $\mathrm{Y}$ & $\mathrm{N}$ & $\mathrm{N}$ & $\mathrm{N}$ & $\mathrm{Y}$ & $Y$ & $\mathrm{~N}$ & $\mathrm{Y}$ & Y & $\mathrm{N}$ & 50 \\
\hline dissust & $\mathrm{N}$ & $\mathrm{N}$ & $\mathrm{N}$ & $\mathrm{Y}$ & N & $\mathrm{N}$ & $\mathrm{N}$ & $\mathrm{N}$ & $\mathrm{N}$ & $\mathrm{N}$ & 10 \\
\hline fear & $\mathrm{N}$ & $\mathrm{N}$ & $\mathrm{Y}$ & $\mathrm{N}$ & $\mathrm{Y}$ & $\mathrm{Y}$ & $\mathrm{N}$ & $\mathrm{N}$ & $\mathrm{N}$ & $\mathrm{N}$ & 30 \\
\hline happy & $\mathrm{N}$ & $\mathrm{Y}$ & $\mathrm{Y}$ & $\mathrm{Y}$ & $\mathrm{N}$ & $\mathrm{Y}$ & $\mathrm{N}$ & $\mathrm{Y}$ & $\mathrm{N}$ & $\mathrm{Y}$ & 60 \\
\hline sad & $\mathrm{N}$ & $\mathrm{Y}$ & $\mathrm{N}$ & $\mathrm{Y}$ & $\mathrm{Y}$ & $\mathrm{Y}$ & $\mathrm{N}$ & $\mathrm{Y}$ & $\mathrm{Y}$ & $\mathrm{N}$ & 60 \\
\hline surprise & $\mathrm{Y}$ & $\mathrm{N}$ & $\mathrm{N}$ & $\mathrm{N}$ & $\mathrm{N}$ & $\mathrm{N}$ & $\mathrm{Y}$ & $\mathrm{N}$ & $\mathrm{N}$ & $\mathrm{N}$ & 20 \\
\hline neutral & $\mathrm{N}$ & $\mathrm{N}$ & $\mathrm{N}$ & $\mathrm{N}$ & $\mathrm{N}$ & $\mathrm{N}$ & $\mathrm{Y}$ & $\mathrm{N}$ & $\mathrm{N}$ & $\mathrm{N}$ & 10 \\
\hline
\end{tabular}

(3) Comparisons of two groups.

From Table 4, the experimental results showed that the recognition rate of facial expressions in autistic children was significantly lower than that in normal children. All the autistic children who participated in the test had a facial recognition rate of less than $60 \%$. Therefore, if the accuracy rate of facial expression diagnosis by the system was less than $60 \%$, the tester would have a tendency to suffer from autism. The lower the recognition rate, the higher the tendency of autism.

Table 4. Comparisons of two groups of children's facial expression recognition rate

\begin{tabular}{|c|c|c|}
\hline Facial cxpression & Normal children group & Autistic children group \\
\hline angry & $80 \%$ & $50 \%$ \\
\hline disgust & $70 \%$ & $10 \%$ \\
\hline fear & $80 \%$ & $30 \%$ \\
\hline happy & $100 \%$ & $60 \%$ \\
\hline sad & $80 \%$ & $60 \%$ \\
\hline surprise & $70 \%$ & $20 \%$ \\
\hline neutral & $90 \%$ & $10 \%$ \\
\hline Average recognition rate $\%$ & $81.4 \%$ & $34.3 \%$ \\
\hline
\end{tabular}

\section{Conclusion}

In this paper, an autism diagnosis system based on deep convolution neural network and expression data is constructed. After testing, it can meet the design requirements of autism diagnosis. The public can download and use the system through the network to diagnose autism conveniently. In addition, we will expand the function of the system, increase the recognition of children's physical movement, and realize the diagnosis of autism from multiple perspectives.

Because the average age of children using and collecting facial expression data is between 3 and 8 years old, the system can recognize children aged 3 to 6 years old. Therefore, through this system, autism can be diagnosed as soon as possible. The earlier the diagnosis and treatment of autism, the better the rehabilitation effect. Therefore, it is of great significance for the treatment of autism.

Because the training samples of the system adopt the international open facial expression database, which contains the facial expression data of children and adults in different countries and regions, the system can diagnose autism for children and adults in different countries and regions.

Of course, the system also needs to be improved through practical use. Next, we will arrange for the system to be tested in a large number of cooperative hospitals. And there are two main tasks to be done. The first is to collect more data of normal and autistic children's facial expressions, improve the recognition effect of the system on children's facial expressions, and establish a special database of children's facial expressions. The second is to improve the system function, according to the results of facial expression 
diagnosis of autistic children for a detailed classification, to distinguish between severe, moderate and mild autism patients, in order to facilitate the treatment of doctors.

This study hopes to be helpful to the diagnosis of autism in remote and underdeveloped areas, so as to promote the early diagnosis and treatment of autistic children and reduce the medical costs and burdens of autistic families and society. Therefore, this study has more important social significance and application value.

\section{References}

[1] Yan Shuqiong,"Experimental study on facial expression processing of autistic children",East China Normal University, 2008.

[2] Segal D L,"Diagnostic and statistical manual of mental disorders (DSM - IV - TR)", The Corsini Encyclopedia of Psychology,2010,pp. 1-3.

[3] Organization W H, The ICD-10 classification of mental and behavioural disorders: clinical descriptions and diagnostic guidelines, Geneva: World Health Organization, 1992.

[4] Wang Guangshuai, Lu Minghui,"Research on educational games for children with autism spectrum disorders", Modern special education,no. 14, 2015, pp. 3840 .

[5] Du Jie,"Research on facial expression recognition based on Kernel ReliefF", Zhengzhou University, 2018.

[6] Li S, Deng W,"Deep facial expression recognition: A survey”, arXiv preprint arXiv:1804.08348, 2018.

[7] Duan Yunfeng, Wu Xiaoli, Jinfeng, "Research progress on etiology and treatment of autism",Chinese Science: Life Science,no. 09, 2015,pp. 820-844.

[8] Vismara L A, Rogers S J,“The Early Start Denver Model”, Journal of Early Intervention, vol. 31, no. 1,2008, pp. 91-108.

[9] Zablotsky B, Black L I, Blumberg S J,"Estimated Prevalence of Children With Diagnosed Developmental Disabilities in the United States, 2014-2016",NCHS Data Brief, no. 291,2017,pp. 1-8.

[10] Wucai Deer Autism Research Institute, Report on the Development of Autism Education and Rehabilitation Industry in China 2, Huaxia Publishing House, 2017.

[11] Wu Xiaoxue, Chen Suyan,"Research progress on quality of life and its influencing factors of primary caregivers for autistic children", General nursing, vol. 16, no. 18,2018 ,pp. 2206-2208.
[12] Yang Y, Wang M,"Employment and Financial Burdens of Families with Preschool-aged Children with Autism",Chinese Journal of Clinical Psychology, vol. 22, no. 2,2014,pp. 295-297, 361.

[13] Singh P, Ghosh S, Nandi S, "Subjective Burden and Depression in Mothers of Children with Autism Spectrum Disorder in India: Moderating Effect of Social Support",Journal of Autism and Developmental Disorders, vol. 47, no. 10,2017,pp. 3097-3111.

[14] Wang Y, Xiao L, Chen R, et al,"Social impairment of children with autism spectrum disorder affects parental quality of life in different ways",Psychiatry Research, no. 266,2018,pp. 168-174.

[15] Liu Yanhong, Huo Wenyao, Hu Xiaoyi,"Summary of research on facial expression recognition of autistic children",Modern special education, no. 8,2015,pp. 35-39.

[16] Yang Jiemin, Huang Xing, Shao Zhi, Yuan Jiajin,"Facial expression sensitivity deficits in patients with autism spectrum disorder: impact of task nature and implications for intervention", Chinese Science: Life Science, vol. 47, no. 04,2017, pp. 443-452.

[17] Shen Xunbing, He Zhifang, Ding Xueping,"Computer facial expression recognition training to improve the facial expression recognition ability of autistic children", Sci-tech horizon, no. 25, 2013, pp. 12-13.

[18] Baron-Cohen S, WheelwrightS, JolliffeIsT, "Is there a "language of the eyes"? Evidence from normal adults, and adults with autism or asperger syndrome", Visual Cognition, vol. 4, no. 3,1997, pp. 311-331.

[19] Wang Hui,"Psychological and behavioral characteristics, diagnosis and evaluation of autistic children", Chinese Journal of Rehabilitation Medicine, vol. 22, no. 9,2007, pp. 853-856.

[20] Hao Yanbin, Wang Fuxing, Xie Ping, et al,"Facial processing characteristics of autism spectrum disorders: meta-analysis of eye movement research",Progress in Psychological Science, no. 1,2018, pp. 26-41.

[21] Lucey P, Cohn J F, Kanade T, et al, “The extended cohn-kanade dataset $\left(\mathrm{ck}^{+}\right)$: A complete dataset for action unit and emotion-specified expression",2010 IEEE Computer Society Conference on Computer Vision and Pattern Recognition-Workshops, 2010. 